\title{
Non-Contact Measurement of Blood Glucose based on Artificial Neural Network
}

\author{
Bagas Satya Dian Nugraha ${ }^{1}$, Faghanie Sugarizka ${ }^{2}$, \\ Fatimah Ken Pratiwi ${ }^{3}$, Risqi Mahardhika ${ }^{4}$, Bambang Sujanarko ${ }^{5}$ \\ ${ }^{1,5}$ Jurusan Teknik Elektro ${ }^{2}$ Program Studi Sistem Informasi ${ }^{3,4}$ Fakultas Kedokteran \\ UNIVERSITAS JEMBER
}

\begin{abstract}
Diabetes mellitus is a disease caused by metabolic disorder that result a lack of insulin in human body. Deficiency of the insulin hormone causes abnormal blood sugar level fluctuation. These conditions should be treated appropriately to prevent the occurrence acute metabolic disorder and chronic complication, so an appropriate treatment can be done by medical personal. This paper develops a non-contact device to measure blood sugar level based on Artificial Neural Network (ANN). ANN in this device convert temperature difference between tragus and antihelix to an index of blood glucose. Tragus and antihelix are the name of two sections of human ear. Using infra red sensor that called thermopile, temperature of these section is measure. So there is no contact between human body and the device. Weight and bias of ANN determine by Back Propagation trained using data from conventional measurement. Experiment result using Matlab Simulink and Peripheral Component Interconnect (PCI) interfacing showed that the device can measured blood glucose and can be used for measurement easier, faster and less intimidating as occurs in conventional measurements.
\end{abstract}

\section{Keywords}

blood glucose level, non-contact measurement, tragus and antihelix, artificial neural network.

\section{INTRODUCTION}

Diabetes (diabetes mellitus) is a group of metabolic syndrome caused by interactions of genetic and environmental factors. Because defects of insulin secretion, insulin action or both, carbohydrates, protein, fat, water and electrolyte metabolism disorders are occurred. Its main common characteristic in clinic is chronic (long term) hyperglycemia [1,2]. Disfunction and failure of various organs are the effects of diabetes mellitus long-term damage. It may present with characteristic symptoms such as thirst, polyuria, blurring of vision, and weight loss. In its most severe forms, ketoacidosis may develop and lead to stupor, coma and, in absence of effective treatment, death [2].

Treatment of hyperglycemic patient is really necessary to do, to eliminate symptoms, to maintain a normal quality of life and work capability, to prevent the occurrence of acute metabolic disorder, and to prevent and delay the occurrence and development of chronic complications. Therefore, measurement of blood sugar levels regularly and periodically is a way to keep sugar levels in balance [3]. Most of methods for blood glucose measurement are invasive. Sample blood from patients is taken and the glucose level is measured, typically by glucose oxidase (GOx) method [2]. Venous whole blood, plasma or serum glucose is tested in hospitals. To maintain the glucose at a desired level, the blood glucose has to be tested several times per day, including at least before and after the three meals and before bed.

All of these methods accurately obtain a blood glucose level, but there are problems and limitations. First, it is inconvenience and very painful to take blood samples multiple times per day. Second, it is costly to use glucose oxidase reagents or test strips either in hospital or at home, which presents a significant financial burden to patients [2]. In addition, self-testing at home may lead to blood contamination and bacterial infection.

Therefore development of a non-invasive type of blood glucose monitoring technologies and devices has been a longterm goal of many research institutions and companies [3]. The main objective of this research is to produce a measurement of blood sugar levels quickly and cheaply. Several non-invasive invention methods are known as reverse iontophoresis and another technique involves transcutaneous harvesting of interstitial fluid from the skin using ultra fine needles, micro-laser drilling, ultrasonic, and disruption chemicals [1-3]. However, the slow response and the attached sensor to the patient become a weakness in blood sugar level measurement.

In this paper will develop a non-contact method that able to measure blood sugar level without touching the patient. The system based on temperature differences between two points on the ear (tragus and anti-helix) [3] that measured by a thermopile. This method supported by artificial neural network (ANN) computing system that has high accuracy and precision [4-6].

\section{SYSTEM DESIGN}

Fig. 1 show the system block diagram of device that used to measure blood glucose in tis research. Basic prinsiple of this system is as follows.

Temperature of tragus and antihelix alternately measure by thermopile. Each scale of these temperature than convert to celcius degree using ANN cicuit. This ANN have two inputs, output of amplifier that amplify thermopile signal and output of amplifier that amplify thermistor signal as compensate environment ambient temperature. These signal enter into computer by PCI interfacing. Weight and Bias of this ANN trained using data that determine by conventional method in the hospital.

After tragus and antihelix temperature obtained, these temperature then convert to blood glucose level using ANN again. Similar as ANN in the previous, this ANN also trained using data that determine by conventional method in the hospital. Finnaly, the blood glucose level displayed on the screen using Matlab GUI. 


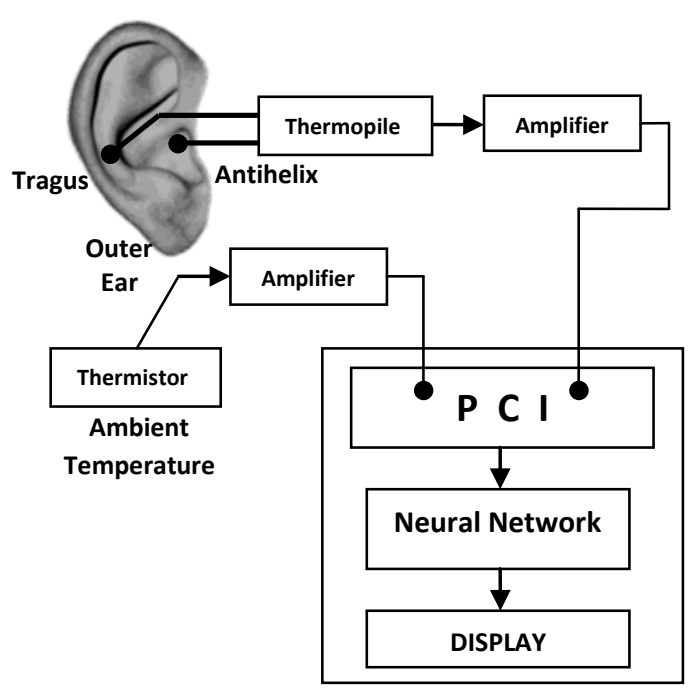

Figure 1. System block diagram

\subsection{Blood Glucose and Tragus-Antihelix Temperature Relation}

Based on the article Larry W. Ishler [3] who explains that the temperature difference between the two regions in the ear (tragus-antihelix) believed to affect changes in blood glucose levels. It can be determined that if the temperature differential decreases, then the blood glucose has increased $1 \mathrm{mg} / \mathrm{dl}$ per approximately $0.024^{\circ} \mathrm{C}$, while if the temperature differential increases, the blood glucose has decreased $1 \mathrm{mg} / \mathrm{dl}$ per approximately $0.024^{\circ} \mathrm{C}$.

\subsection{Thermopile and Amplification}

\section{Circuit}

Based on the thermoelectric effect, the thermopile can be used as a heat sensor to measure thermal radiation. A thermopile is made of thermocouple junction pairs connected electrically in series [7]. A thermocouple consists of two dissimilar conductors in contact, which can produce a voltage when heated. The voltage produced is dependent on the difference of temperature of the junction to other parts of the circuit. Thermocouples are a widely used type of temperature sensor for measurement and control and can also be used to convert a temperature gradient into electricity [8].

In this research, thermopile used as a temperature detector of tragus and antihelix. In principle, the thermopile has the characteristic of any increase temperature will cause voltage rise. However, the voltage on the body or ear temperature measurement has very small differences that needed amplifying circuit, so that the voltages must amplify.

This amplification circuit consists of two operational amplifier (op amp) [9]. The two op amp in this circuit used as a non-inverting amplifier. Fig. 2 shows the circuit diagram of a non-inverting amplifier. The input voltage $V_{\text {in }}$ is applied to the non-inverting op amp input. A voltage divider consisting of resistors $R_{F}$ and $R_{i}$ connects from the output node to the inverting input. The circuit is called a non-inverting amplifier because its voltage gain is positive. This means that if the input voltage is increasing or going positive, the output voltage will also be increasing going positive.

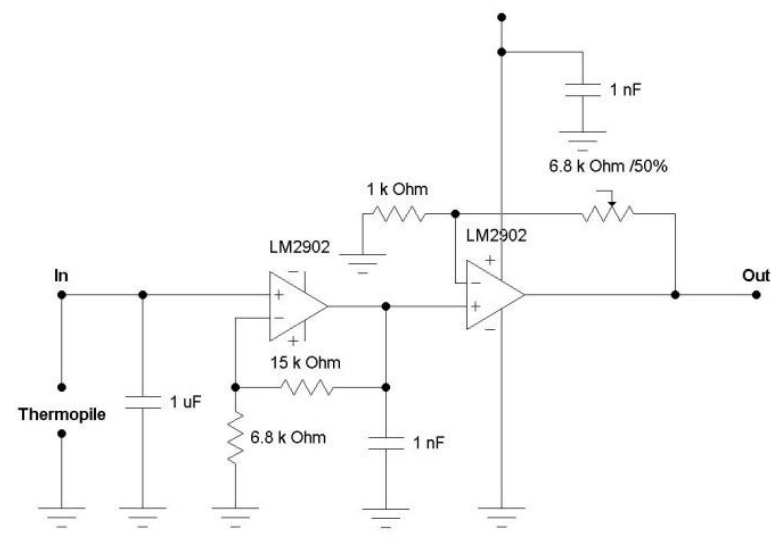

Figure 2. Thermopile Amplifier

\subsection{Thermistor and Amplification Circuit}

A thermistor is a special type of semiconductor that varies in resistance in a predictable way as temperature is changed $[8,10]$. Thermistors can be classified into two types: If the resistance increases with increasing temperature, the device is called a positive temperature coefficient (PTC). If the resistance decreases with increasing temperature, the device is called a negative temperature coefficient (NTC) thermistor. In this case we use the type of NTC thermistor [10].

Thermistor is used for the compensation of an environment temperature. According to the variation of an environment temperature, the output of thermopile detector is also varying. It is therefore need to compensate this temperature performance of thermopile detector. The thermopile detectors are using a thermistor for the compensation of an environment temperature. In this invention, the resistant value of thermistor is converted to voltage value to make it easier to compensate with the thermopile output. Fig. 3 shows the amplifier circuit of thermistor.

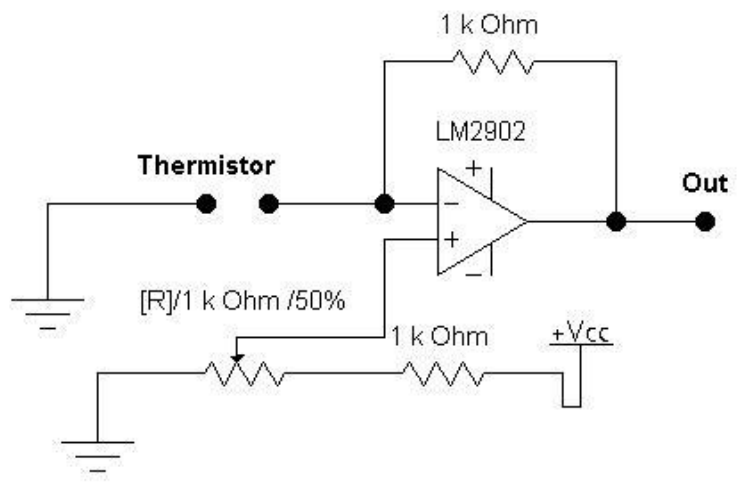

Figure 3. Thermistor Amplifier

\subsection{PCI, Interfacing Program and Real- Time Works Matlab Simulink}

PCI is a local computer bus for attaching hardware devices in a computer. The PCI bus supports the functions found on a processor bus, but in a standardized format that is independent of any particular processor. Devices connected to the bus appear to the processor to be connected directly to the 
processor bus, and are assigned addresses in the processor's address space [11].

PCI Express (Peripheral Component Interconnect Express), officially abbreviated as PCIe, is a high-speed serial computer expansion bus standard designed to replace the older PCI, PCI-X, and AGP bus standards. PCIe has numerous improvements over the aforementioned bus standards, including higher maximum system bus throughput, lower I/O pin count and smaller physical footprint, better performancescaling for bus devices, a more detailed error detection and reporting mechanism (Advanced Error Reporting (AER ${ }^{[]}$), and native hot-plug functionality. More recent revisions of the PCIe standard support hardware I/O virtualization.

PCI Express is used as the data communication and interface between the sensor and the computer device. The result of thermopile and thermistor is analog which then converted into digital data. The conversion of analog to digital data facilitates the processing data on computer, so that the application of artificial neural network (ANN) will also be easier.

MATLAB (matrix laboratory) is a numerical computing environment and fourth-generation programming language. Developed by MathWorks, MATLAB allows matrix manipulations, plotting of functions and data, implementation of algorithms, creation of user interfaces, and interfacing with programs written in other languages, including $\mathrm{C}, \mathrm{C}++$, Java, and Fortran [12].

Simulink is a software package for modeling, simulating, and analyzing dynamical systems. It supports linear and nonlinear systems, modeled in continuous time, sampled time, or a hybrid of the two. Systems can also be multirate.

\subsection{Neural Network Design}

Artificial Neural Network (ANN) is an interconnected group of natural or artificial neurons that uses a mathematical or computational model for information processing based on a connectionistic approach to computation [4-6]. In most cases an ANN is an adaptive system that changes its structure based on external or internal information that flows through the network. Artificial Neural Network is a non-linier data processing system which has high accuracy and precision. It is possible to provide accurate and precise blood glucose measurement. In this case, artificial neural network (ANN) used as a processing data system from temperature result that detected by thermopile sensor and thermistor.

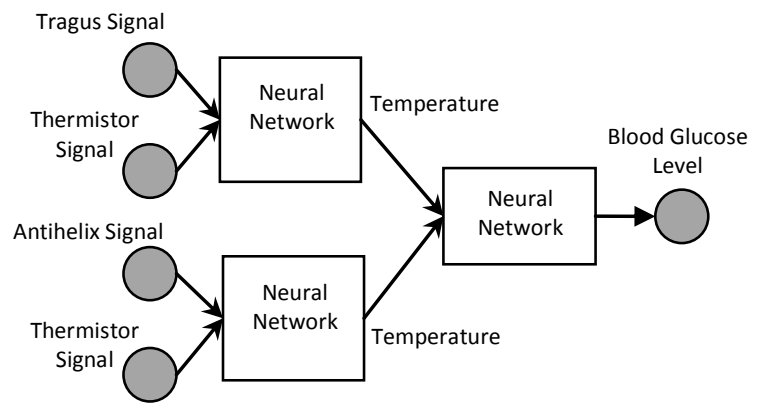

Figure 2. Neural Network Structure

The construction of non-contact blood glucose measurement involve two steps of neural network computation. First step is the processing of thermopile and thermistor measurement which converted into degrees centigrade. This is necessary because the result of measurement sensors is formed in voltage (volts), so that needs to change into temperature unit to facilitate further measurement. The second step is the acquisition value of blood sugar levels by measuring the ear temperature at tragus and antihelix region obtained from the first neural network process using these sensors.

The first neural network structure is formed by the training using the data that obtained from conventional temperature measurement, amplified thermopile voltage, and amplified thermistor voltage. Especially on thermopile, sensor will divide by two times measurement, i.e. tragus region measurement and antihelix region measurement. The training result formed in tragus and antihelix temperature will be the data for the next blood glucose neural network training.

The tragus and antihelix temperature that have been obtained from the first neural network training is the data for the second neural network training that will result blood glucose level value. This blood glucose level value is established by the training process using tragus and antihelix temperature and blood glucose level that are conventionally taken in hospital.

\section{RESULT AND DISCUSSION}

Within 7 patients of Soebandi Hospital at Jember Indonesia, the result of glucose measurement using Gox and the system show at Table 1 .

Table 1. Device Measurement Result

\begin{tabular}{|c|c|c|c|c|}
\hline Object & $\begin{array}{c}\text { Tragus } \\
\text { Temp. } \\
\left({ }^{\circ} \mathrm{C}\right)\end{array}$ & $\begin{array}{c}\text { Antihelix } \\
\text { Temp. } \\
\left({ }^{\circ} \mathrm{C}\right)\end{array}$ & $\begin{array}{c}\text { Blood } \\
\text { Glucose } \\
\text { with GOx } \\
(\mathrm{mg} / \mathrm{dL})\end{array}$ & $\begin{array}{c}\text { Blood } \\
\text { Glucose } \\
\text { with NN } \\
(\mathrm{mg} / \mathrm{dL})\end{array}$ \\
\hline I & 33.4 & 31.2 & 100 & 107 \\
\hline II & 33.1 & 29.3 & 125 & 121 \\
\hline III & 33.5 & 30.2 & 115 & 112 \\
\hline IV & 34.3 & 31.9 & 120 & 110 \\
\hline V & 32.4 & 30.9 & 160 & 163 \\
\hline VI & 35.9 & 33.8 & 155 & 160 \\
\hline VII & 32.1 & 30.7 & 170 & 166 \\
\hline
\end{tabular}

Based on table 1, comparison between measurement using neural network device with conventional measurement have a small difference. The data shows error percentage of $0.98 \%$ $1.07 \%$. With this value of the error, we can obtained statement that blood glucose measurement using non-contact system with artificial neural network has given good and accurate result.

\section{CONCLUSION}

Non-contact measurement of Glucose based on Artificial Intelegent can build and have error percentage of $0.98 \%$ $1.07 \%$. This method can prevent painful of patient and can avoid blood contamination and bacterial infection.

\section{ACKNOWLEDGMENT}

This work was supported by Program Kreativitas Mahasiswa (PKM), Direktorat Jendral Pendidikan Tinggi, Departemen Pendidikan dan Kebudayaan Republik Indonesia. 


\section{REFERENCES}

[1] Definition and diagnosis of diabetes mellitus and intermediate hyperglycemia: report of a WHO/IDF consultation. Geneva: World Health Organization. 2006.

[2] Julio Raba and Horacio A. Mottola (1995). Glucose Oxidase as an Analytical Reagent. Critical Reviews in Analytical Chemistry.

[3] Larry W. Ishler and Erie, USA Patent. 2003. NonInvasive Blood Glucose Moitoring Sistem.

[4] Ganesan, N. Application of Neural Networks in Diagnosing Cancer Disease Using Demographic Data (PDF). International Journal of Computer Applications. Retrieved 2013-7-15.

[5] Bottaci, Leonardo. Artificial Neural Networks Applied to Outcome Prediction for Colorectal Cancer Patients in Separate Institutions (PDF). The Lancet. Retrieved 20137-15.
[6] http://uhaweb.hartford.edu/compsci/neural-networksLearning.html. Retrieved 2013-7-16.

[7] GE Measurement and Control. Application Note: Thermopile IR Sensor Applications (PDF). 2012.

[8] Practical Temperature Measurements (PDF). Agilent Application Note. Agilent Semiconductor. Retrieved 2013-7-16.

[9] Texas Instruments. Understanding Basic Analog: Ideal Op Amps (Application Report SLAA068A)(PDF). 2000. Retrieved 2013-7-18.

[10] Thermistors \& Thermocouples:Matching the Tool to the Task in Thermal Validation (PDF). Journal of Validation Technology. Retrieved 2013-7-15.

[11] http://www.webopedia.com/TERM/P/PCI.html Retrieved 2013-7-17.

[12] Simulink: Dynamic System Simulation for Matlab (PDF).http://www.mathworks.com/products/matlab/?s_ci d=wiki_matlab_15. Retrieved 2013-7-16. 